\title{
A Simple Test for Causality in Volatility
}

\author{
Chia-Lin Chang ${ }^{1}$ and Michael McAleer ${ }^{2,3,4,5, *}$ \\ 1 Department of Applied Economics, Department of Finance, National Chung Hsing University, \\ 40227 Taichung City, Taiwan; changchialin@nchu.edu.tw \\ 2 Department of Quantitative Finance, National Tsing Hua University, 30013 Hsinchu City, Taiwan \\ 3 Econometric Institute, Erasmus School of Economics, Erasmus University Rotterdam, 3062 PA Rotterdam, \\ The Netherlands \\ 4 Department of Quantitative Economics, Complutense University of Madrid, 28040 Madrid, Spain \\ 5 Institute of Advanced Sciences, Yokohama National University, 240-8501 Yokohama, Japan \\ * Correspondence: michael.mcaleer@gmail.com; Tel.: +886-3-571-5131
}

Academic Editor: Marc S. Paolella

Received: 3 January 2017; Accepted: 16 March 2017; Published: 20 March 2017

\begin{abstract}
An early development in testing for causality (technically, Granger non-causality) in the conditional variance (or volatility) associated with financial returns was the portmanteau statistic for non-causality in the variance of Cheng and $\mathrm{Ng}$ (1996). A subsequent development was the Lagrange Multiplier (LM) test of non-causality in the conditional variance by Hafner and Herwartz (2006), who provided simulation results to show that their LM test was more powerful than the portmanteau statistic for sample sizes of 1000 and 4000 observations. While the LM test for causality proposed by Hafner and Herwartz (2006) is an interesting and useful development, it is nonetheless arbitrary. In particular, the specification on which the LM test is based does not rely on an underlying stochastic process, so the alternative hypothesis is also arbitrary, which can affect the power of the test. The purpose of the paper is to derive a simple test for causality in volatility that provides regularity conditions arising from the underlying stochastic process, namely a random coefficient autoregressive process, and a test for which the (quasi-) maximum likelihood estimates have valid asymptotic properties under the null hypothesis of non-causality. The simple test is intuitively appealing as it is based on an underlying stochastic process, is sympathetic to Granger's $(1969,1988)$ notion of time series predictability, is easy to implement, and has a regularity condition that is not available in the LM test.
\end{abstract}

Keywords: random coefficient stochastic process; simple test; Granger non-causality; regularity conditions; asymptotic properties; conditional volatility

JEL Classification: C22; C32; C52; C58

\section{Introduction}

Although there have been many practical applications of testing causality (technically, Granger non-causality) of the conditional mean, especially in economics (see, for example, Geweke (1984) [1], Hoover (2001) [2], Granger et al. (1986) [3], Comte and Lieberman (2000) [4], Hafner and Herwartz (2008) [5], Lee and Yang (2014) [6], Candelon and Topkavi (2016) [7], and Corsi et al. (2015) [8]), there have been fewer applications of testing for causality in conditional higher moments, especially the variance or volatility associated with financial returns.

An early development was the portmanteau statistic of non-causality in the variance of Cheng and $\mathrm{Ng}$ (1996) [9]. A subsequent development was the Lagrange Multiplier (LM) test of non-causality in the conditional variance (technically, in the conditional volatility) by Hafner and Herwartz (2006) [10], 
who provided simulation results to show that their LM test was more powerful than the portmanteau statistic for sample sizes of 1000 and 4000 observations.

This result should not be especially surprising, as LM tests are generally more powerful than portmanteau tests, wherein the null hypothesis is well specified but the alternative is not, in order to capture a wide range of departures from the null. On the other hand, the LM test is intended to have high power of a null hypothesis when the true value of the parameter is close to that given under the null.

While the LM test for causality proposed by Hafner and Herwartz (2006) [10] is an interesting and useful development, it is nonetheless arbitrary. In particular, the specification on which the LM test is based does not rely on an underlying stochastic process, so that the alternative hypothesis is also arbitrary, which can affect the power of the test.

The purpose of the paper is to derive a simple intuitively appealing test for causality in volatility that is sympathetic to Granger's $(1969,1988)[11,12]$ notion of predictability using a VAR time series model, provides regularity conditions that arise from the underlying stochastic process [11,12], namely a random coefficient autoregressive process, is easy to implement, and has a regularity condition that is not available in the LM test of Hafner and Herwartz (2006) [10], which is based on an arbitrary specification. This paper also aims to derive a test for which the (quasi-) maximum likelihood estimates have valid asymptotic properties under the null hypothesis of non-causality.

The plan of the paper is as follows. Section 2 provides a simple test for causality in volatility that is sympathetic to Granger's $(1969,1988)[11,12]$ notion of predictability using a VAR time series model. Section 3 compares the new test algebraically with the LM test of Hafner and Herwartz (2006) [10]. Section 4 gives some concluding comments.

\section{A Simple Test for Causality in Volatility}

Consider the conditional mean of financial returns for commodity $i$, as follows:

$$
y_{i t}=E\left(y_{i t} \mid I_{t-1}\right)+\varepsilon_{i t}, i=1,2, \ldots, m
$$

where the returns, $y_{i t}=\Delta \log P_{i t}$, represent the log-difference in financial commodity prices, $P_{t}, I_{t-1}$ is the information set for all financial assets at time $t-1, E\left(y_{i t} \mid I_{t-1}\right)$ is the conditional expectation of returns, and $\varepsilon_{i t}$ is a conditionally heteroskedastic error term.

In what follows, we derive a simple test for causality in volatility that provides regularity conditions arising from the underlying stochastic process. The simple test is intuitively appealing as it is sympathetic to Granger's $(1969,1988)[11,12]$ notion of time series predictability, and is easy to implement.

In order to derive conditional volatility specifications, it is necessary to specify the stochastic processes underlying the returns shocks, $\varepsilon_{i t}$, which may be written as a random coefficient autoregressive process, as follows:

$$
\varepsilon_{i t}=\phi_{i t} \varepsilon_{i t-1}+\phi_{j t} \varepsilon_{j t-1}+\eta_{i t}, i \neq j
$$

where

$$
\begin{aligned}
\phi_{i t} & \sim \operatorname{iid}\left(0, \alpha_{i}\right), \alpha_{i} \geq 0 \\
\phi_{j t} & \sim \operatorname{iid}\left(0, \alpha_{j}\right), \alpha_{j} \geq 0 \\
\eta_{i t} & \sim \operatorname{iid}\left(0, \omega_{i}\right), \omega_{i} \geq 0 .
\end{aligned}
$$

$h_{i t}$ is the conditional volatility of financial asset $i$.

Setting $\phi_{j t}=0$ in (2) leads to a random coefficient autoregressive model, namely,

$$
\varepsilon_{i t}=\phi_{i t} \varepsilon_{i t-1}+\eta_{i t}
$$


which gives the conditional volatility of financial asset $i$ as an ARCH process (see Engle (1982) [13]):

$$
E\left(\varepsilon_{i t}^{2} \mid I_{t-1}\right) \equiv h_{i t}=\omega_{i}+\alpha_{i} \varepsilon_{i t-1}^{2} .
$$

The stochastic process given in Equation (2) incorporates causality, so that the null hypothesis of non-causality holds when $\phi_{j t}=0$, which is equivalent to $\alpha_{j}=0$. The stochastic process can be extended to asymmetric conditional volatility models (see, for example, McAleer (2014) [14]), and to give higher-order lags and a larger number of alternative commodities, namely up to $m-1$. However, the symmetric bivariate process considered here is sufficient to focus the key ideas associated with testing for causality in volatility.

The conditional volatility arising from the bivariate random coefficient autoregressive process in Equation (2) is given as

$$
E\left(\varepsilon_{i t}^{2} \mid I_{t-1}\right) \equiv h_{i t}=\omega_{i}+\alpha_{i} \varepsilon_{i t-1}^{2}+\alpha_{j} \varepsilon_{j t-1}^{2} .
$$

Equation (5) is the basis for a simple test of causality in volatility. An arbitrary addition of first-order lags of both $h_{i t}$ and $h_{j t}$ leads to a conditional specification that gives a simple test for causality in volatility that is sympathetic to Granger's $(1969,1988)[11,12]$ notion of predictability, namely,

$$
h_{i t}=\omega_{i}+\alpha_{i} \varepsilon_{i t-1}^{2}+\alpha_{j} \varepsilon_{j t-1}^{2}+\beta_{i} h_{i t-1}+\beta_{j} h_{j t-1}
$$

in which $\alpha_{i} \geq 0, \alpha_{j} \geq 0, \beta_{i} \in(-1,1)$, and $\beta_{j} \in(-1,1)$. The model in Equation (6) is a $\operatorname{GARCH}(1,1)$ model for commodity $i$ (see Bollerslev (1986) [15], with an extension to incorporate both short- and long-run volatility spillovers from commodity $j$.

As the stochastic process in Equation (2), and hence also in Equation (3), follows a random coefficient autoregressive process, and satisfies the invertibility condition, under normality (or non-normality) of the random errors, the maximum likelihood estimators (or quasi- maximum likelihood estimators) of the parameters will be consistent and asymptotically normal under the null hypothesis of non-causality in volatility. Ling and McAleer (2003) [16] and McAleer et al. (2008) [17] provide general proofs of the asymptotic properties of multivariate conditional volatility models based on satisfying the regularity conditions in Jeantheau (1998) [18] for consistency (log-normal or multivariate log-normal condition), and in Theorem 4.1.3 in Amemiya (1985) [19] for asymptotic normality.

A Lagrange Multiplier (LM), Likelihood Ratio, or Wald test for causality, or of Granger non-causality, is a test of the null hypothesis,

$$
H_{0}: \alpha_{j}=\beta_{j}=0
$$

against the alternative hypothesis,

$$
H_{1}: \alpha_{j}>0, \beta_{j} \neq 0 .
$$

Hafner and Herwartz (2006) [10] recommend the LM test statistic, as it requires estimation under the null hypothesis of non-causality. The appropriate test statistic follows an asymptotic $\chi^{2}(2)$ distribution under the null hypothesis of non-causality. Note that the test is one-sided for $\alpha_{j}$ as it cannot be negative, though it can be conducted as a two-tailed test, as in the LM test of Hafner and Herwartz (2006) [10].

It is worth noting that the model of conditional volatility in Equation (6) holds under the alternative hypotheses, as it is a valid conditional volatility equation arising from the bivariate random coefficient autoregressive process in Equation (2). 


\section{Algebraic Comparison with the Hafner and Herwartz LM Test}

Using the notation of this paper, the LM test of Hafner and Herwartz (2006) [10] is based on the specification given as

$$
\varepsilon_{i t}=\eta_{i t} \sqrt{h_{i t}} \sqrt{g_{j t}}
$$

where $g_{j t}$ is, in effect, a GARCH $(1,1)$ model for commodity $j$, namely,

$$
g_{j t}=\omega_{j}+\alpha_{j} \varepsilon_{j t-1}^{2}+\beta_{j} h_{j t-1} .
$$

In Equation (10), $\omega_{j}$ can be set arbitrarily to unity, and $g_{j t}$ can be replaced by $h_{j t}$ without loss of generality. The LM test is a test of the null hypothesis in Equation (7), which is equivalent to $g_{j t}=1$, against the alternative hypothesis,

$$
H_{1}: \alpha_{j} \neq \beta_{j} \neq 0
$$

which is a two-sided LM test statistic, and is asymptotically distributed as $\chi^{2}(2)$ under the null hypothesis of non-causality in volatility.

It is worth noting that, although the test of the null against the alternative based on Equation (9) is statistically valid, it does not have a clear underlying stochastic process, as it is a product of a definition of the standardized shocks of commodity $i, \eta_{i t}$,

$$
\varepsilon_{i t}=\eta_{i t} \sqrt{h_{i t}}
$$

and, as stated above, the conditional volatility of commodity $j, g_{j t}$, which can be replaced by $h_{j t}$, without loss of generality.

Moreover, the conditional expectation of $\varepsilon_{i t}^{2}$, which is the conditional volatility of $\varepsilon_{i t}$ in Equation (9), is given by

$$
h_{i t}=h_{i t} g_{j t}
$$

which holds only under the null hypothesis in Equation (7), in which $g_{j t}=1$, whereas the specification underlying the simple test given in Equation (6) holds under both the null and the alternative hypotheses.

\section{Conclusions}

An early development in testing for causality in conditional variance (technically the conditional volatility) associated with financial returns was the portmanteau statistic for non-causality in the variance of Cheng and $\mathrm{Ng}$ (1996) [9]. A subsequent development was the LM test of non-causality in the conditional variance by Hafner and Herwartz (2006) [10], who provided simulation results to show that their LM test was more powerful than the portmanteau statistic.

Although the LM test for causality proposed by Hafner and Herwartz (2006) [10] is interesting and a useful development, it is nonetheless arbitrary. In particular, the specification on which the LM test is based does not rely on an underlying stochastic process, so the alternative hypothesis is also arbitrary, which can affect the power of the test.

The purpose of the paper was to derive a simple test for causality in volatility that is sympathetic to Granger's $(1969,1988)[11,12]$ notion of predictability using a VAR time series model and provides regularity conditions that arise from the underlying stochastic process, namely a random coefficient autoregressive process, and a test for which the (quasi-) maximum likelihood estimates have valid asymptotic properties under the null hypothesis of non-causality in volatility.

The simple test is intuitively appealing, as it is based on an underlying stochastic process, is sympathetic to Granger's notion of time series predictability, is easy to implement, and has a regularity condition that is not available in the LM test. 
Acknowledgments: The authors are most grateful to two referees for very helpful comments and suggestions. For financial support, the first author wishes to thank the National Science Council, Ministry of Science and Technology (MOST), Taiwan, and the second author acknowledges the Australian Research Council and the National Science Council, Ministry of Science and Technology (MOST), Taiwan.

Author Contributions: M.M. provided the algebraic results, but both authors contributed equally to the final version of the paper.

Conflicts of Interest: The authors declare no conflict of interest.

\section{References}

1. Geweke, J. Inference and causality in economic time series. In Handbook of Econometrics, Vol. 2; Griliches, Z., Intriligator, M.D., Eds.; Elsevier: Amsterdam, The Netherlands, 1984; Volume 2, pp. 1101-1144.

2. Hoover, K.D. Causality in Macroeconomics; Cambridge University Press: Cambridge, UK, 2001.

3. Granger, C.W.J.; Robins, R.P.; Engle, R.F. Wholesale retail prices: Bivariate time series modeling with forecastable error variances. In Model Reliability; MIT Press: Boston, MA, USA, 1986; pp. 1-17.

4. Comte, F.; Lieberman, O. Second-order noncausality in multivariate GARCH processes. J. Time Ser. Anal. 2000, 21, 535-557. [CrossRef]

5. Hafner, C.M.; Herwartz, H. Testing for causality in variance using multivariate GARCH models. Ann. Econ. Stat. 2008, 89, 215-241. [CrossRef]

6. Lee, T.; Yang, W. Granger-causality in quantiles between financial markets: Using copula approach. Int. Rev. Financ. Anal. 2014, 33, 70-78. [CrossRef]

7. Candelon, B.; Topkavi, S. A nonparametric test for Granger causality in distribution with application to financial contagion. J. Bus. Econ. Stat. 2016, 34, 240-253. [CrossRef]

8. Corsi, F.; Lillo, F.; Pirino, D. Measuring Flight-to-Quality with Granger-Causality Tail Risk Networks. 2015. Available online: http:/ /dx.doi.org/10.2139/ssrn.2576078 (accessed on 17 February 2017).

9. Cheung, Y.W.; Ng, L.K. A causality in variance test and its application to financial market prices. J. Econ. 1996, 72, 33-48. [CrossRef]

10. Hafner, C.M.; Herwartz, H. A Lagrange multiplier test for causality in variance. Econ. Lett. 2006, 93, $137-141$. [CrossRef]

11. Granger, C.W.J. Investigating causal relations by econometric models and cross spectral methods. Econometrica 1969, 37, 424-438. [CrossRef]

12. Granger, C.W.J. Some recent developments in a concept of causality. J. Econ. 1988, 39, 199-211. [CrossRef]

13. Engle, R.F. Autoregressive conditional heteroscedasticity with estimates of the variance of United Kingdom inflation. Econometrica 1982, 50, 987-1007. [CrossRef]

14. McAleer, M. Asymmetry and leverage in conditional volatility models. Econometrics 2014, 2, 145-150. [CrossRef]

15. Bollerslev, T. Generalised autoregressive conditional heteroscedasticity. J. Econ. 1986, 31, 307-327.

16. Ling, S.; McAleer, M. Asymptotic theory for a vector ARMA-GARCH model. Econ. Theory 2003, 19, $278-308$. [CrossRef]

17. McAleer, M.; Chan, F.; Hoti, S.; Lieberman, O. Generalized autoregressive conditional correlation. Econ. Theory 2008, 24, 1554-1583. [CrossRef]

18. Jeantheau, T. Strong consistency of estimators for multivariate ARCH models. Econ. Theory 1998, 14, 70-86. [CrossRef]

19. Amemiya, T. Advanced Econometrics; Harvard University Press: Cambridge, MA, USA, 1985.

(c) 2017 by the authors. Licensee MDPI, Basel, Switzerland. This article is an open access article distributed under the terms and conditions of the Creative Commons Attribution (CC BY) license (http:/ / creativecommons.org/licenses/by/4.0/). 